\title{
A Unified Sequent Calculus for Focused Proofs
}

\author{
Chuck Liang \\ Hofstra University \\ Department of Computer Science \\ Hempstead, NY, USA \\ Email: Chuck.C.Liang@hofstra.edu
}

\author{
Dale Miller \\ INRIA Saclay - Ille-de-France \\ LIX/Ecole Polytechnique \\ Palaiseau, France \\ Email: dale.miller@inria.fr
}

\begin{abstract}
We present a compact sequent calculus LKU for classical logic organized around the concept of polarization. Focused sequent calculi for classical logic, intuitionistic logic, and multiplicative-additive linear logic are derived as fragments of LKU by increasing the sensitivity of specialized structural rules to polarity information. We develop a unified, streamlined framework for proving cut-elimination in the various fragments. Furthermore, each sublogic can interact with other fragments through cut. We also consider the possibility of introducing classical-linear hybrid logics.
\end{abstract}

Keywords-Proof theory; focused proof systems; linear logic

\section{INTRODUCTION}

While it is well-known how to describe proof systems for intuitionistic and linear logics as restrictions on structural rules and formula within a classical logic proof system, these logics are usually studied separately. Girard merged these three logics into a unified sequent calculus called LU [1] in such a way that these three logics appear as fragments that can interact via the cut rule. Central to LU is a classification of formulas according to one of three polarities, which are used to identify the formulas on which structural rules apply.

There are potentially many computer science applications that could mix classical, intuitionistic, and linear logics. For example, a logic program might be mostly classical logic but in certain situations require the dynamics of linear logic (e.g., multiset rewriting). Type systems for a programming language can be intuitionistic when dealing with functions but classical when dealing with control operators [2]. With these kinds of computer science applications, one needs to establish adequacy results that confirm that formal proofs using the logic specification really capture what is intended in the application. When proving such adequacy results over sequent calculus proofs, one discovers that there can be enormous numbers of sequent proofs, most of which differ only in minor ways. For this reason, adequacy results are usually based on the normal form of sequent calculus proofs known as focused proofs. In the area of logic programming, such proofs present backchaining as an atomic action [3]. In type systems, focused proofs provide a canonical form for terms.

Focused proof systems, such as Andreoli's proof system for linear logic (see [4] and Figure 1) organize proofs into two phases: one phase contains the invertible inference rules while the other phase contains the (possibly) non-invertible rules. The two polarities of negative (invertible) and positive (non-invertible) arise from these two phases. The polarity notions used in LU can be seen as compatible with those used in focusing proof systems. However, LU is not focused. We have developed LKU as a focused proof system for a unifying approach to logic. The differences between LKU and LU, however, are not limited to focusing. In particular, LU can be described by a translation to linear logic (except at the level of atoms). The LKU proof system (see Figure 3) is a classical logic and there is no translation of LKU proofs into linear logic proofs: instead, each of its fragments may require a different translation. As in Gentzen's original systems, intuitionistic (and linear) logic can be seen as subsystems of LKU with restrictions on the use of structural rules.

The proof system LKU contains a rich set of logical connectives (a merging of the connectives in linear, intuitionistic, and classical logics) and each connective has one inference rule. This stands in sharp contrast to LU where several connectives have a large number of introduction rules. On the other hand, LU provides a small and fixed set of structural rules while LKU has a larger set of structural rules (being a focusing proof system causes some growth in these rules). In LKU, the meaning of a connective, such as $\oplus$ and 78 , is determined not only by their (usual) introduction rule but also by the sensitivity of the structural rules to their polarity. By adjusting this sensitivity we can use the various symbols of LKU to derive focusing systems for classical logic, intuitionistic logic, MALL (multiplicative additive linear logic), and other interesting fragments of these logics. Since these fragments are based on formulas containing the same set of connectives, it is possible for these fragments to interact through cut elimination. Placing more emphasis on structural rules also seems to be valuable since much of the effort in designing focusing proof systems is centered on what structural rules they should include. For example, one can have systems that focus on a unique formula or on multiple formulas [5]. One can insist that an asynchronous phase terminates when all asynchronous formulas are removed or allow it to terminate before they are all removed. 
In applications of focusing proofs to tabled deduction [6], once an atom is proved, there are good reasons to switch its polarity from negative to positive, but in such a setting, one should restrict the "reaction" rules to not be applicable for positive atoms (in order to avoid reproving atoms). The LKU system should be a framework for studying a range of possible restrictions to the structural rules. While fragments of LKU will be defined by imposing restrictions on the structural rules, all fragments share the same set of nine introduction rules. Such uniformity simplifies proving cutelimination for fragments.

We do not claim for LKU all that LU promises. In particular, although never fully explained or further studied, LU leaves open the possibility of allowing hybrid formulas that use connectives from multiple logics without restriction, e.g., $(A \otimes B) \wedge C$. While such a possibility is not within the scope of LKU, we consider limited classical-linear hybrid logics in Section VII and as future work in Section VIII.

\section{The Focusing Proof Systems LLF And LKF}

There are many examples of proof systems in literature that exhibit characteristics of focusing to one degree or another. These include, for example, uniform proofs [3], "polarized" proof systems LJT/LJQ [7], [8] and $\mathrm{LK}_{p}^{\eta}$ [9], as well as the more recent "mixed polarization" proof system $\lambda$ RCC [10]. Andreoli [4] identified focusing as arising from a duality between invertible and non-invertible inference rules and presented the "two-phase" proof system LLF presented in Figure 1.

A literal is an atomic formula or the negation of an atomic formula. Connectives of linear logic are either asynchronous, negative $(\&, 28, \forall, ?)$ or synchronous, positive $(\oplus, \otimes, \exists, !)$. Atoms are assigned arbitrary polarity. LLF uses two kinds of sequents. In the sequent $\vdash \Gamma: \Delta \Uparrow L$, the "zones" $\Gamma$ and $\Delta$ are multisets. In the original system $L$ is a list, but it is also valid to consider $L$ as a multiset. This sequent encodes the usual one-sided sequent $\vdash$ ? $\Gamma, \Delta, L$. The $\Gamma$ to the left of the : is the classical or unbounded context, while the $\Delta$ is the linear or bounded context. This sequent will also satisfy the invariant that $\Delta$ contains only literals and synchronous formulas. In the sequent $\Gamma: \Delta \Downarrow F$, the zone $\Gamma$ is a multiset of formulas, $\Delta$ is a multiset of literals and synchronous formulas, and $F$ is a single formula. The use of these two zones replaces the need for explicit weakening and contraction rules.

In the LLF proof system in Figure 1, inference rules involving the $\Uparrow$ belong to the asynchronous phase and rules involving the $\Downarrow$ belong to the synchronous phase. The $I_{1}$ and $I_{2}$ rules are the initial rules. Of the structural rules, the reaction rules $R \Uparrow$ and $R \Downarrow$ and the decision rules $D_{1}$ and $D_{2}$ stand out: they will collectively be called the structural rules. Some formulations of focusing, e.g., [9], [11], avoid a presentation with two arrows in favor of careful descriptions of when a sequent proof is actually focused.

$$
\begin{aligned}
& \frac{\Gamma: \Delta \Uparrow L}{\Gamma: \Delta \Uparrow \perp, L} \quad \frac{\Gamma: \Delta \Uparrow F, G, L}{\Gamma: \Delta \Uparrow F \not 8 G, L} \quad \frac{\Gamma, F: \Delta \Uparrow L}{\Gamma: \Delta \Uparrow ? F, L} \\
& \overline{\Gamma: \Delta \Uparrow \top, L} \quad \frac{\Gamma: \Delta \Uparrow F, L \quad \Gamma: \Delta \Uparrow G, L}{\Gamma: \Delta \Uparrow F \& G, L} \quad \frac{\Gamma: \Delta \Uparrow B[y / x], L}{\Gamma: \Delta \Uparrow \forall x . B, L} \\
& \frac{\Gamma: \Delta, F \Uparrow L}{\Gamma: \Delta \Uparrow F, L}[R \Uparrow] \text { provided that } \mathrm{F} \text { is not asynchronous } \\
& \overline{\Gamma: \Downarrow \Downarrow 1} \quad \frac{\Gamma: \Delta_{1} \Downarrow F \quad \Gamma: \Delta_{2} \Downarrow G}{\Gamma: \Delta_{1}, \Delta_{2} \Downarrow F \otimes G} \quad \frac{\Gamma: \cdot \Uparrow F}{\Gamma: \cdot \Downarrow ! F} \\
& \frac{\Gamma: \Delta \Downarrow F_{1}}{\Gamma: \Delta \Downarrow F_{1} \oplus F_{2}} \quad \frac{\Gamma: \Delta \Downarrow F_{2}}{\Gamma: \Delta \Downarrow F_{1} \oplus F_{2}} \quad \frac{\Gamma: \Delta \Downarrow B[t / x]}{\Gamma: \Delta \Downarrow \exists x . B}
\end{aligned}
$$$$
\frac{\Gamma: \Delta \Uparrow F}{\Gamma: \Delta \Downarrow F}[R \Downarrow] \quad \text { provided that } F \text { is either asynchronous or a }
$$$$
\text { If } K \text { a positive literal: } \overline{\Gamma: K^{\perp} \Downarrow K}\left[I_{1}\right] \quad \overline{\Gamma, K^{\perp}: \Downarrow K}\left[I_{2}\right]
$$

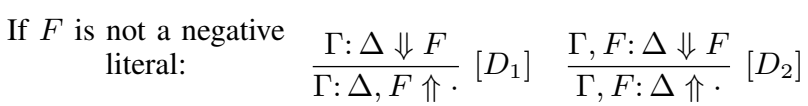

Figure 1. The focused proof system LLF

In LLF, the structural rules and the initial rules $I_{1}$ and $I_{2}$ are the rules that are directly sensitive to polarity information: these rules show that it is polarity that drives focusing. In fact, if the polarity-related side conditions for these rules are removed, we are left with a rather convoluted version of an unfocused sequent calculus for linear logic where one would be able to switch between the $\Downarrow$ and the $\Uparrow$ states without regard to change in polarity. Notice also that the rules for the exponential operators ? and ! behave less like other introduction rules and more like the reaction rules $R \Uparrow$ and $R \Downarrow$.

Structural rules in the style of LLF will play a critical role in our unified sequent calculus. In fact, our project here is first to present a rich set of LLF-like structural rules and then to investigate different subsets of those structural rules to see how they account for different proof systems (for, say, intuitionistic or linear logics).

LLF-style focused systems have also been adapted to classical and intuitionistic logic. In [12], the authors presented the focused intuitionistic sequent calculus LJF that can be seen as an LU-inspired translation of intuitionistic logic into linear logic. That paper also presented the focused classical sequent calculus LKF that was inspired by a double-negation translation into LJF (similar to Girard's LC [13]). The system LKF is given in Figure 2 (a one-side presentation of LJF is given in Figure 5). Here, $P$ is positive, $N$ is negative, $C$ is a positive formula or a negative literal, $\Theta$ consists of positive formulas and negative literals, and $x$ is not free in $\Theta$, $\Gamma$. Focused and unfocused sequents have the form $\mapsto[\Theta], A$ and $\vdash[\Theta], \Gamma$, respectively.

The additive and multiplicative versions of conjunction and disjunction are available in both LKF and LU. In LU, 


$$
\begin{aligned}
& \frac{\vdash[\Theta, C], \Gamma}{\vdash[\Theta], \Gamma, C}[] \frac{\mapsto[P, \Theta], P}{\vdash[P, \Theta]} \text { Focus } \frac{\vdash[\Theta], N}{\mapsto[\Theta], N} \text { Release } \\
& \overline{\mapsto[\neg P, \Theta], P} I D^{+} \text {, atom } P \underset{\mapsto[N, \Theta], \neg N}{ } I D^{-} \text {, atom } N \\
& \overline{\mapsto[\Theta], \mathcal{T}} \quad \overline{\vdash[\Theta], \Gamma, \neg \mathcal{F}} \quad \frac{\vdash[\Theta], \Gamma}{\vdash[\Theta], \Gamma, \neg \mathcal{T}} \\
& \frac{\vdash[\Theta], \Gamma, A \quad \vdash[\Theta], \Gamma, B}{\vdash[\Theta], \Gamma, A \wedge^{-} B} \quad \frac{\vdash[\Theta], \Gamma, A, B}{\vdash[\Theta], \Gamma, A \vee B} \\
& \frac{\vdash[\Theta], \Gamma, A}{\vdash[\Theta], \Gamma, \forall x A} \quad \frac{\mapsto[\Theta], A \mapsto[\Theta], B}{\mapsto[\Theta], A \wedge^{+} B} \\
& \frac{\mapsto[\Theta], A_{i}}{\mapsto[\Theta], A_{1} \vee^{+} A_{2}} \quad \frac{\mapsto[\Theta], A[t / x]}{\mapsto[\Theta], \exists x A}
\end{aligned}
$$

Figure 2. The Focused Proof System LKF

one must examine the polarity the connective's arguments to determine the additive/multiplicative nature of that connective. In LKF, the top-level logical symbol provides this information: $\wedge^{-}$and $V^{+}$are additive while $\wedge^{+}$and $V^{-}$are multiplicative. The difference between the two conjunctions and two disjunctions lies in the focused proofs that they admit: they are, however, provably equivalent. In contrast, the linear connectives $\otimes$ and $\&$ are not provably equivalent.

While LKF inherits the structural rules of LLF, the reaction rules Release and [] of LKF correspond not to $R \Downarrow$ and $R \Uparrow$ but to the ! and ? introduction rules of LLF. The decision rule $D_{2}$ in LLF corresponds directly to the LKF rule Focus: both embody an explicit contraction. There is, however, an important difference between these two proof systems regarding the formulas that are contracted. In LKF (and LJF), formulas selected for focus (and thus subjected to contraction) are always positive. In LLF, however, the ? introduction rule stops asynchronous decomposition and so asynchronous formulas are also subject to contraction. The restriction of contraction to only positive formulas is an important characteristic of LKF and prompts us to adopt this feature to our unified system. In fact, we exchange the ability to represent full linear logic for the benefits of a system that is better behaved with respect to focusing, and which can still accommodate classical, intuitionistic, and multiplicative-additive linear logic. This simplification of LKF also leads to a more direct proof of cut-elimination (without the need for Gentzen's mix rule [14]).

\section{LKU}

Central to the LKU proof system, found in Figure 3, are the four polarities +2 (positive classical), +1 (positive linear), -1 (negative linear), and -2 (negative classical). Atomic formulas are assigned polarities from this set. Other formulas derive their polarity from their top-level connective as follows: $\wedge^{+}, v^{+}, \exists, 1,0$ are given polarity $+2 ; \otimes, \oplus, \Sigma$ are given polarity $+1 ; \not 28, \&, \Pi$ are given polarity -1 ; and $\wedge^{-}, \vee^{-}, \forall, \top, \perp$ are given polarity -2 . Negation $\left(A^{\perp}\right)$ is defined by the following De Morgan dualities: $\otimes / \not \mathcal{Q}, \oplus / \&$, $\wedge^{+} / \vee^{-}, \vee^{+} / \wedge^{-}, \Sigma / \Pi, \exists / \forall, 1 / \perp, \top / 0, A / A^{\perp}$ for literals A. The dual polarity of +1 is -1 and the dual of +2 is -2 . Formulas are assumed to be in negation normal form.

A large number of connectives are included in LKU. The polarity scheme of LKU is superficially similar to that of LU. The "neutral" polarity of LU is here divided into the +1 and -1 polarities. Unlike $\mathrm{LU}$, the distinctions between the connectives is not fixed within the unified logic. The introduction rules make no distinction between the linear and classical versions of each connective: the notation $\left[\otimes \mid \wedge^{+}\right]$means that the rule is applicable to both connectives. LKU can be divided into two principal components: the introduction rules, which are invariant for all fragments, and the collection of initial, decision, and reaction rules (collectively called "structural" rules here), which can be restricted to define sublogics. The structural rules are further divided between the "level-1" and "level-2" rules.

LKU sequents of the form $\vdash \Gamma: \Delta \Downarrow B$ and $\vdash \Gamma: \Delta \Uparrow \Theta$ and will always satisfy the invariant that $\Gamma$ and $\Delta$ contain only positive formulas or negative literals. As a consequence, the only possible instances of the two initial rules $I_{1}$ and $I_{2}$ (Figure 3) will be such that $P$ is a positive literal.

As given, LKU can only be called classical logic. The four connectives for conjunction, $\wedge^{+}, \wedge^{-}, \otimes$, and \& are all provably equivalent, as are the four for disjunction and the pairs of quantifiers and units. The structural rules are only sensitive to the positive/negative distinction and not to the linear/classical distinction. If we removed even this basic level of sensitivity to polarity, then we are left with a verbose version of the unfocused LK. Clearly, the inference rules of LKU are sound with respect to classical logic. The classical completeness of LKU follows from the completeness of LKF [12], which it contains.

A focusing phase of a proof ends in either a reaction rule, $R_{1} \Downarrow$ or $R_{2} \Downarrow$, or in an initial rule $I_{1}$ or $I_{2}$. The ? and ! rules of LLF reappear in LKU as the level-2 reaction rules $R_{2} \Uparrow$ and $R_{2} \Downarrow$. As given, $R_{2} \Downarrow$ is subsumed by $R_{1} \Downarrow$ : their distinction will become clear when we consider sublogics. Both the $R_{1} \Uparrow$ and $R_{2} \Uparrow$ rules exclude asynchronous formulas, as does the $D_{2}$ rule. This divergence from LLF means that we will not be able to represent full-linear logic for reasons explained in the previous section. These restrictions are similar to those of polarized linear logic [15]. In LKU the role of the exponential operators is replaced entirely by polarity information. If we relaxed these restrictions and allowed $R_{2} \Uparrow$ and $D_{2}$ to be applicable for asynchronous formulas, then clearly every LLF proof can be mimicked. Although a unified logic that accommodates full linear logic is certainly an interesting topic (see Section VIII), the restriction that we adopt is also worthy of separate study.

Fragments of LKU are defined by restricting the structural 


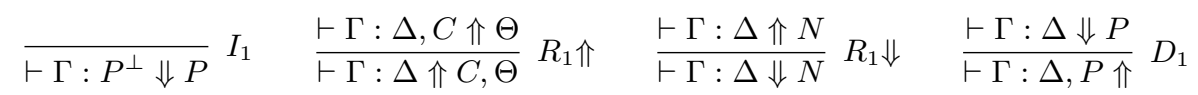

$$
\begin{aligned}
& \overline{\vdash \Gamma, P^{\perp}: \Downarrow P} I_{2} \quad \frac{\vdash C, \Gamma: \Delta \Uparrow \Theta}{\vdash \Gamma: \Delta \Uparrow C, \Theta} R_{2} \Uparrow \quad \frac{\vdash \Gamma: \Uparrow N}{\vdash \Gamma: \Downarrow N} R_{2} \Downarrow \quad \frac{\vdash P, \Gamma: \Delta \Downarrow P}{\vdash P, \Gamma: \Delta \Uparrow} D_{2} \\
& \frac{\vdash \Gamma: \Delta \Uparrow A, B, \Theta}{\vdash \Gamma: \Delta \Uparrow A\left[\not \mathcal{B} \mid \vee^{-}\right] B, \Theta}\left[\not 8 \mid \vee^{-}\right] \quad \frac{\vdash \Gamma: \Delta \Uparrow \Theta}{\vdash \Gamma: \Delta \Uparrow \perp, \Theta} \perp \quad \frac{\vdash \Gamma: \Delta \Uparrow A, \Theta \vdash \Gamma: \Delta \Uparrow B, \Theta}{\vdash \Gamma: \Delta \Uparrow A\left[\& \mid \wedge^{-}\right] B, \Theta}\left[\& \mid \wedge^{-}\right] \quad \overline{\vdash \Gamma: \Delta \Uparrow \top, \Theta}{ }^{\top}
\end{aligned}
$$

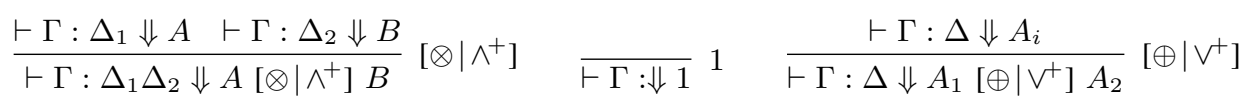

$$
\begin{aligned}
& \frac{\vdash \Gamma: \Delta \Downarrow A[t / y]}{\vdash \Gamma: \Delta \Downarrow[\Sigma \mid \exists] y \cdot A}[\Sigma \mid \exists] \frac{\vdash \Gamma: \Delta \Uparrow A, \Theta}{\vdash \Gamma: \Delta \Uparrow[\Pi \mid \forall] x \cdot A, \Theta}[\Pi \mid \forall]
\end{aligned}
$$

The rules for Initial, Reaction, and Decision as well as three introduction rules for the units, eight rules for the binary connectives, and four rules for the quantifiers. Here, $P$ is positive $(+2$ or +1$), N$ is negative $(-2$ or -1$)$, and $C$ is a positive formula or negative literal.

Figure 3. The Unified Focusing Sequent Calculus LKU

rules and possibly also the forms of formulas used. Not all fragments, however, can be called "logics" (see Section VII). Assume that end-sequents of LKU all have the form $\vdash$ : $\Gamma$. The following fragments are immediate.

Focused MALL. If we forbid all uses of the level-2 structural rules and only allow $I_{1}, D_{1}, R_{1} \Uparrow$, and $R_{1} \Downarrow$, then the resulting system is essentially the same as LLF restricted to the MALL fragment (but with quantifiers). We shall call this fragment MALLF. Note that "forbidding level-2 rules" is not the same as forbidding the $+2 /-2$ polarities: the units $0,1, \perp$ and $T$ are all still accounted for in MALLF. In fact, we still retain all the connectives of LKU, but symbols such as $\wedge^{+}$and $\otimes$ will both be interpreted as linear connectives.

LKF. If we forbid all the level-1 rules and only allowed the level-2 structural rules then we arrive at a more conventional sequent calculus for classical logic, one that's similar to LKF. Symbols such as $\otimes$ and $\not 8$ are retained but they will have the same meaning as their classical counterparts.

Retaining seemingly redundant symbols facilitates the communication between different fragments of LKU through cut, which is difficult to formalize if the fragments use disjoint sets of connectives.

\section{The Intuitionistic Fragment}

Intuitionistic logic appears as a linear-classical hybrid fragment within LKU and, as a result, that fragment provides a focused proof system for intuitionistic logic. We illustrate this by showing how the LJF proof system of [12] can be seen as a fragment of LKU. Since LJF is itself a framework for describing a range of focused proof systems (e.g., LJT

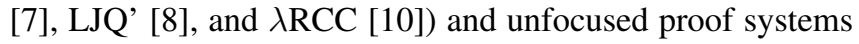
(e.g., LJ [14]) for intuitionistic logic, describing LJF is a good test of LKU's expressiveness.

Formulas in LJF are "annotated" intuitionistic formulas: that is, atomic formulas are assigned an arbitrary but fixed polarity (either positive or negative) and conjunctions are annotated as being either additive $\wedge^{-}$or multiplicative $\wedge^{+}$. The LJF proof system is a two-sided sequent calculus using sequents of the following styles. The premises and conclusion of invertible inference rules use sequents of the form $[\Gamma], \Theta \longrightarrow R$ : such sequents lack focus. Dually, the premises and conclusion of non-invertible inference rules use sequents such as $[\Gamma] \stackrel{L}{\longrightarrow}[R]$, which provides a "left-focus" formula $L$ or sequents such as $[\Gamma]-{ }_{R} \rightarrow$, which provides a "right-focus" formula $R$. A set of "structural rules" are provided in LJF that mix sequents of both kinds.

LJF formulas are mapped into LKU formulas using the two functions $[\cdot]^{R}$ and $[\cdot]^{L}$ defined in Figure 4 . This is a shallow, syntactic mapping of intuitionistic connectives (whose proof rules are described using two-sided sequents) to classical connectives (whose proof rules are described using one-sided sequents). That is, a left-occurrence of the intuitionistic $\supset$ is exactly the same as (a right-occurrences of) the LKU connective $\otimes$. Positive LJF atoms are assigned polarity +2 in LKU while negative LJF atoms are assigned polarity -1 in LKU. Formulas in the range of $[\cdot]^{R}$ are called essentially right intuitionistic formulas (they have polarity +2 or -1$)$ and formulas in the range of $[\cdot]^{L}$ are called essentially left intuitionistic formulas (they have polarity -2 or +1 ). Notice that $R$ is an essentially right intuitionistic formula if and only if the negation normal form of $R^{\perp}$ is an essentially left intuitionistic formula.

The usual symbols of linear logic are used to define the negative intuitionistic connectives. The left-hand side of an essentially right implication is essentially left (and vice versa) and is given a classical treatment by the reaction rules, thus mimicking the usual linear-logic interpretation of intuitionistic implication as $! A \multimap B$. As with LKF, the LJF fragment contains both positive and negative connectives for conjunction: $\wedge^{+}$and \& respectively on the right $\left(V^{-}\right.$and $\oplus$ on the left). However, there is only the positive disjunction $\mathrm{V}^{+}\left(\wedge^{-}\right.$on the left), with $\not 8$ only used in the representation 


$$
\begin{aligned}
& {\left[B \wedge^{-} C\right]^{R}=[B]^{R} \&[C]^{R} \quad\left[B \wedge^{+} C\right]^{R}=[B]^{R} \wedge^{+}[C]^{R}} \\
& {[B \supset C]^{R}=[B]^{L} \not 8[C]^{R} \quad[B \vee C]^{R}=[B]^{R} \vee^{+}[C]^{R}} \\
& {[\forall x \cdot B]^{R}=\Pi x \cdot[B]^{R} \quad[\exists x \cdot B]^{R}=\exists x \cdot[B]^{R}} \\
& {\left[B \wedge^{-} C\right]^{L}=[B]^{L} \oplus[C]^{L} \quad\left[B \wedge^{+} C\right]^{L}=[B]^{L} \vee^{-}[C]^{L}} \\
& {[B \supset C]^{L}=[B]^{R} \otimes[C]^{L} \quad[B \vee C]^{L}=[B]^{L} \wedge^{-}[C]^{L}} \\
& {[\forall x . B]^{L}=\Sigma x \cdot[B]^{L} \quad[\exists x \cdot B]^{L}=\forall x \cdot[B]^{L}}
\end{aligned}
$$

For atomic $A,[A]^{R}=A$ and $[A]^{L}=A^{\perp}$.

Figure 4. Mapping LJF formulas into LKU.

of intuitionistic implication. Intuitionistic negation $\sim A$ is defined as $A^{\perp}>80$ when appearing essentially right. (For a minimal logic treatment of negation, replace 0 in the language with some designated +2 atom.)

While we do not present the full LJF proof system (see [12]) to save space, it can be seen as a simple fragment within LKU. In particular, if one restricts the structural rules of LKU to be those given in Figure 5, we are left with a one-sided sequent presentation of LJF. To illustrate how two sided inference rules for intuitionistic logic can be represented in the one-sided, focused setting of LKU, consider the additive and multiplicative versions of the conjunction-left rule in (unfocused) LJ:

$$
\frac{A_{i}, \Gamma \vdash C}{A_{1} \wedge A_{2}, \Gamma \vdash C} \text { and } \frac{A_{1}, A_{2}, \Gamma \vdash C}{A_{1} \wedge A_{2}, \Gamma \vdash C} .
$$

These inference rules correspond to the focused LKU rules

$$
\frac{\vdash \Gamma^{\perp}: C \Downarrow A_{i}^{\perp}}{\vdash \Gamma^{\perp}: C \Downarrow A_{1}^{\perp} \oplus A_{2}^{\perp}} \quad \text { and } \quad \frac{\vdash \Gamma^{\perp}: C \Uparrow A_{1}^{\perp}, A_{2}^{\perp}}{\vdash \Gamma^{\perp}: C \Uparrow A_{1}^{\perp} \vee^{-} A_{2}^{\perp}} .
$$

For the reader familiar with LJF [12], the two-sided LJF sequents correspond to the one-sided LKU sequents as follows:

$$
\begin{aligned}
{[\Gamma], \Theta \longrightarrow R } & \longleftrightarrow & \vdash[\Gamma]^{L}: \Uparrow[\Theta]^{L},[R]^{R} \\
{[\Gamma], \Theta \longrightarrow[R] } & \longleftrightarrow & \vdash[\Gamma]^{L}:[R]^{R} \Uparrow[\Theta]^{L} \\
{[\Gamma]-{ }_{R} \longrightarrow } & \longleftrightarrow & \vdash[\Gamma]^{L}: \cdot \Downarrow[R]^{R} \\
{[\Gamma] \stackrel{L}{\longrightarrow}[R] } & \longleftrightarrow & \vdash[\Gamma]^{L}:[R]^{R} \Downarrow[L]^{L}
\end{aligned}
$$

The original structural rules of LJF and those in Figure 5 correspond as follow: $L f \leftrightarrow D_{2}, R f \leftrightarrow D_{1}, R_{l} \leftrightarrow R_{1} \Downarrow$, $R_{r} \leftrightarrow R_{2} \Downarrow,[]_{l} \leftrightarrow R_{2} \Uparrow,[]_{r} \leftrightarrow R_{1} \Uparrow$.

In the following we only consider LJF in its form as a fragment of LKU. Of the structural rules of LJF, $I_{1}, R_{1} \Downarrow$, $R_{2} \Uparrow$, and $D_{2}$ can be called "left rules" while $I_{2}, R_{2} \Downarrow, R_{1} \Uparrow$, and $D_{1}$ are the right rules.

Observe that the $R_{1} \Uparrow$ and $R_{1} \Downarrow$ rules allow only one essentially right formula inside the linear context of an LJF sequent. If we are interested only in mapping complete LJF proofs to intuitionistic proofs, then this restriction is not necessary: the single-conclusion condition is essentially already enforced by other rules such as $R_{2} \Downarrow, I_{1}$, and $I_{2}$. When building a proof from the bottom-up, malformed

$$
\begin{gathered}
\frac{\vdash \Gamma: Q^{\perp} \Downarrow Q}{\vdash 1} I_{1} \frac{\vdash \Gamma: C \Uparrow \Theta}{\vdash \Gamma: \Uparrow C, \Theta} R_{1} \Uparrow \frac{\vdash \Gamma: C \Uparrow \mathcal{N}}{\vdash \Gamma: C \Downarrow \mathcal{N}} R_{1} \Downarrow \\
\frac{\vdash \Gamma: \Delta \Downarrow \mathcal{P}}{\vdash \Gamma: \Delta, \mathcal{P} \Uparrow} D_{1} \quad \frac{\vdash \Gamma, \mathcal{Q}^{\perp}: \Downarrow \mathcal{Q}}{\vdash 2} \frac{\vdash D, \Gamma: \Delta \Uparrow \Theta}{\vdash \Gamma: \Delta \Uparrow D, \Theta} R_{2} \Uparrow \\
\frac{\vdash \Gamma: \Uparrow N}{\vdash \Gamma: \Downarrow N} R_{2} \Downarrow \frac{\vdash P, \Gamma: \Delta \Downarrow P}{\vdash P, \Gamma: \Delta \Uparrow} D_{2}
\end{gathered}
$$

$Q:+1$ atom, $C:+2$ formula or -1 atom, $\mathcal{N}:-2$ formula,

$\mathcal{P}:+2$ formula, $\mathcal{Q}:+2$ atom, $D:+1$ formula or -2 literal, $N$ : -1 formula, $P:+1$ formula

Figure 5. LJF as a Fragment of LKU

sequents, i.e., those with multiple essentially right formulas, will be rejected by the initial rules if not sooner.

The stronger restrictions for the $R_{1} \Uparrow$ and $R_{1} \Downarrow$ rules allow us to establish the stronger correspondence between open proofs as well. In LJF, malformed sequents could appear as a consequence of splitting the context when applying the $\otimes$ rule. The essentially-left occurrence of an implication $A \supset$ $B$ has the form $A \otimes B^{\perp}$ where $A$ is essentially right and $B^{\perp}$ essentially left. The implication-left rule of LJ thus appears in the form

$$
\frac{\vdash \Gamma^{\perp}: \Downarrow A \vdash \Gamma^{\perp}: C \Downarrow B^{\perp}}{\vdash \Gamma^{\perp}: C \Downarrow A \otimes B^{\perp}} \otimes
$$

But it is also possible to split the context so as to have $\vdash \Gamma^{\perp}: C \Downarrow A$, which is a sequent with two essentially right formulas. The reaction rules of LJF are designed, however, to reject such a malformed sequent at the end of a focusing $(\Downarrow)$ phase. Such a phase must end in either a reaction or an initial rule. In an incomplete proof structure, there could be occurrences of malformed sequents inside the synchronous phases of proofs, but we shall only consider completed phases as marking the boundary of inference rules: what defines a focused proof is not what happens in the details of each synchronous or asynchronous phase but what happens at the borders of such phases. Each synchronous or asynchronous phase can be thought of as the introduction of a synthesized connective; that is to say a single introduction rule. A border sequent of LJF will be either an axiom or have the form $\vdash \Gamma^{\perp}: C \Uparrow$, which corresponds to a well-formed intuitionistic sequent. Without the explicit restriction to one formula in the level-1 reaction rules, malformed sequents may survive across focusing phases.

Thus if we strictly use only polarity information in restricting the structural rules, we can achieve a weak form of full-completeness. With the stronger forms of the rules as presented, the local structure of even partial intuitionistic proofs are preserved.

There is, however, one scenario in which a malformed sequent may also appear as part of a complete LJF proof. When considering full intuitionistic logic, as opposed to 


$$
\begin{aligned}
& \frac{\vdash \Gamma: Q^{\perp} \Downarrow Q}{\vdash} I_{1} \frac{\vdash \Gamma: C \Uparrow \Theta}{\vdash \Gamma: \Uparrow C, \Theta} R_{1} \Uparrow \frac{\vdash P, \Gamma: \Delta \Uparrow \Theta}{\vdash \Gamma: \Delta \Uparrow P, \Theta} R_{2} \Uparrow \\
& \frac{\vdash \Gamma: \Uparrow N}{\vdash \Gamma: \Downarrow N} R_{2} \Downarrow \frac{\vdash P, \Gamma: \Delta \Downarrow P}{\vdash P, \Gamma: \Delta \Uparrow} D_{2} \\
& Q:+1 \text { literal, } C:-1 \text { atom, } P:+1 \text { formula, } N:-1 \text { formula }
\end{aligned}
$$

Figure 6. The nLJF fragment of LKU.

minimal logic, the intuitionistic context may be inconsistent. That is to say the $T$ rule ( 0 on the left) may appear in a proof. This problem is likewise encountered by LU and several other works that encodes intuitionistic logic into linear logic (including LJF). To resolve this problem we must show that even in such situations there is a LJF proof that corresponds to a well-formed LJ proof. Such an argument relies on cutelimination (see Section V).

This treatment of intuitionistic logic in LKU is similar to that in LU with two differences. First, LU is a twosided sequent calculus in order to accommodate intuitionistic logic. The richness of polarity information in LKU replaces the need for a two-sided system: the polarity of a formula unambiguously determines its essentially left or right status. (Of course, one may still prefer a two-sided system for readability.) Second, it is equally valid in LKU to use the $-2 /+1$ polarities for essentially right formulas and $+2 /-1$ for the left ones by altering the restrictions on the structural rules.

The Negative Intuitionistic Fragment: There is a significant fragment of LJF where the problem with context splitting in the $\otimes$ rule does not appear. We shall call this fragment the negative intuitionistic fragment $n L J F$ and it corresponds to the neutral intuitionistic fragment of LU. The structural rules that correspond to $\mathrm{nLJF}$ are found in Figure 6 . In this fragment, essentially right formulas have only polarity -1 and essentially left formulas have only polarity +1 . In an essentially left implication $A \otimes B^{\perp}, A$ will have -1 polarity, which means that the appearance of a malformed sequent $\vdash \Gamma^{\perp}: C \Downarrow A$ will immediately invoke the $R_{2} \Downarrow$ rule, which fails because the linear context is not empty.

\section{Cut Elimination}

In order to claim that a fragment of LKU is, in fact, a logic, one needs to show that the result of eliminating a cut between two proofs in the given fragment yields a proof still in that fragment. In this section, we illustrate how to prove such a result. We later examine what kinds of cutfree proofs can be derived when different fragments of LKU cross-cut. The LKU framework provides a uniform structure to cut-elimination arguments. Since the introduction rules are shared by all the fragments, the permutation of cut above introductions can be demonstrated just once. Furthermore, instead of considering individual rules, we can define the following relations to characterize the structure of complete synchronous and asynchronous phases. For convenience, we write $\Gamma \Gamma^{\prime}$ to denote the multiset union of $\Gamma$ and $\Gamma^{\prime}$.

Definition 1: Let $\uparrow$ and $\downarrow$ represent relations between formulas and multisets of formulas defined as follows: $\circ A \uparrow\{A\}$ if $A$ is a negative literal or positive. $\circ \perp \uparrow\{\}$.

$\circ\left(A\left[\not \mathcal{P} \mid \vee^{-}\right] B\right) \uparrow \Phi \Phi^{\prime}$ if $A \uparrow \Phi$ and $B \uparrow \Phi^{\prime}$.

$\circ\left(A\left[\& \mid \wedge^{-}\right] B\right) \uparrow \Phi$ if $A \uparrow \Phi$.

$\circ\left(A\left[\& \mid \wedge^{-}\right] B\right) \uparrow \Phi^{\prime}$ if $B \uparrow \Phi^{\prime}$.

$\circ A \downarrow\{A\}$ if $A$ is a positive literal or negative.

$\circ 1 \downarrow\{\}$.

$\circ\left(A\left[\otimes \mid \wedge^{+}\right] B\right) \downarrow \Psi \Psi^{\prime}$ if $A \downarrow \Psi$ and $B \downarrow \Psi^{\prime}$.

$\circ\left(A\left[\oplus \mid \mathrm{V}^{+}\right] B\right) \downarrow \Psi$ if $A \downarrow \Psi$.

$\circ\left(A\left[\oplus \mid \vee^{+}\right] B\right) \downarrow \Psi^{\prime}$ if $B \downarrow \Psi^{\prime}$.

(First-order quantification $[\Pi \mid \forall],[\Sigma \mid \exists]$ can be treated similarly.)

Using these dual relations, we can study how cuts permute only where it matters the most: at the borders between positive and negative focusing phases where the rules of reaction and decision come into play. In MALL, the distributive laws can be used to provide the following normal forms for all positive and negative synthetic connectives:

$$
\oplus_{i \in I}\left(\otimes_{j \in J_{i}} N_{i j}\right) \quad \&_{i \in I}\left(\not_{j \in J_{i}} P_{i j}\right),
$$

where $I$ and $J_{i}$ (for $i \in I$ ) are finite set of indices and $N_{i j}$ denotes a negative formula or a literal and $P_{i j}$ denotes a positive formula or a literal. Using the notation above, the following are satisfied:

$$
\begin{aligned}
& \oplus_{i \in I}\left(\otimes_{j \in J_{i}} N_{i j}\right) \downarrow\left\{N_{i j} \mid j \in J_{i}\right\} \quad(i \in I) \\
& \&_{i \in I}\left(\not{P}_{j \in J_{i}} P_{i j}\right) \uparrow\left\{P_{i j} \mid j \in J_{i}\right\} \quad(i \in I)
\end{aligned}
$$

Thus, the $\downarrow$ selects the premises for a possible introduction rule of a positive synthetic connective while the $\uparrow$ selects a possible premise for the introduction rule of a negative synthetic connective. While normal forms for synthetic connectives are equivalent to using the $\downarrow$ and $\uparrow$ within MALL, one does not expect that similar distributive laws hold for all fragments of LKU and, as a consequence, normal forms for synthetic connectives might be hard to write down. For this reason, we employ the notation using arrows since they provide natural and immediate descriptions of the introduction rules for synthetic connectives in all of LKU.

Lemmas 2 through 4 below are all proved by induction on the structure of formulas.

Lemma 2: Given a formula $R$, let $\Phi_{1}, \ldots, \Phi_{m}$ be multisets such that $R \uparrow \Phi_{1}, \ldots, R \uparrow \Phi_{m}$ and if $R \uparrow \Phi$ then $\Phi=\Phi_{i}$ for some unique $1 \leq i \leq m$. Every cut-free proof of $\vdash \Gamma: \Delta \Uparrow R, \Theta$ is of the form

$$
\frac{\vdash \Gamma \Phi_{1}^{1}: \Delta \Phi_{1}^{2} \Uparrow \Theta}{\vdots} \ldots \frac{\vdash \Gamma \Phi_{m}^{1}: \Delta \Phi_{m}^{2} \Uparrow \Theta}{\vdots}
$$


such that $\Phi_{i}^{1} \Phi_{i}^{2}=\Phi_{i}$ for each $1 \leq i \leq m$. For every $\Phi_{i}$ such that $\Phi_{i}=\Phi_{i}^{1} \Phi_{i}^{2}$, if

$$
\vdash \Gamma \Phi_{1}^{1}: \Delta \Phi_{1}^{2} \Uparrow \Theta, \ldots, \vdash \Gamma \Phi_{m}^{1}: \Delta \Phi_{m}^{2} \Uparrow \Theta
$$

are all cut-free provable, then $\vdash \Gamma: \Delta \Uparrow R, \Theta$ is also cutfree provable.

The splitting of $\Phi_{i}$ into $\Phi_{i}^{1}$ and $\Phi_{i}^{2}$ represents the choice between $R_{1} \Uparrow$ and $R_{2} \Uparrow$. The above lemma does not specify how $\Phi_{i}^{1}$ and $\Phi_{i}^{2}$ are split: e.g., $\Phi_{i}^{2}$ may be empty. Given a fragment of LKU, we can specialize this lemma by being more specific as to how the multiset is split between the linear and classical contexts. That is, we can deduce specialized versions of this lemma for each fragment from the general lemma above. In the MALLF fragment, $\Phi_{i}^{1}$ must be empty. In the intuitionistic LJF fragment, $\Phi_{i}^{1}$ consists of essentially left formulas and $\Phi_{i}^{2}$ consists of at most one essentially right formula (see lemma 7 below).

The dual lemma for $\downarrow$ is the following.

Lemma 3: Let $R \downarrow\left\{a_{1}, \ldots, a_{n}\right\}$ and assume that $\vdash \Gamma$ : $\Delta_{1} \Downarrow a_{1}, \ldots, \vdash \Gamma: \Delta_{n} \Downarrow a_{n}$ are all cut-free provable. Then $\vdash \Gamma: \Delta_{1} \ldots \Delta_{n} \Downarrow R$ is also cut-free provable. Furthermore, every cut-free proof of $\vdash \Gamma: \Delta_{1} \ldots \Delta_{n} \Downarrow R$ is of the form

$$
\frac{\frac{\vdash \Gamma: \Delta_{1} \Downarrow a_{1}}{\vdots} \ldots \frac{\vdash \Gamma: \Delta_{n} \Downarrow a_{n}}{\vdots}}{\frac{1}{\vdash \Gamma: \Delta_{1} \ldots \Delta_{n} \Downarrow R}}
$$

where $R \downarrow\left\{a_{1}, \ldots, a_{n}\right\}$.

Cut-elimination follows from the following lemma.

Lemma 4: $R \uparrow\left\{a_{1}, \ldots, a_{n}\right\}$ if and only if $R^{\perp} \downarrow$ $\left\{a_{1}^{\perp}, \ldots, a_{n}^{\perp}\right\}$.

The inductive measure for the cut-elimination proof is the usual lexicographical ordering on the size of the cut formula and the heights of subproofs. In a focused proof, the height of a proof can be taken as the maximum number of alternating asynchronous-synchronous phases (i.e., the number of $D_{1}$ and $D_{2}$ rules) along a path to a leaf.

Theorem 5: The cut rule

$$
\frac{\vdash \Gamma: \Uparrow A, \Theta \vdash \Gamma^{\prime}: \Uparrow A^{\perp}, \Theta^{\prime}}{\vdash \Gamma \Gamma^{\prime}: \Uparrow \Theta \Theta^{\prime}} c u t
$$

can be eliminated in LKF. Similarly, the cut rule

$$
\frac{\vdash: \Delta \Uparrow A, \Theta \quad \vdash: \Delta^{\prime} \Uparrow A^{\perp}, \Theta^{\prime}}{\vdash: \Delta \Delta^{\prime} \Uparrow \Theta \Theta^{\prime}} c u t
$$

can be eliminated in MALLF. Finally, the cut rule

$$
\frac{\vdash \Gamma^{\perp}: \Uparrow A \vdash \Delta^{\perp}: \Omega \Uparrow A^{\perp}, \Theta}{\vdash \Gamma^{\perp} \Delta^{\perp}: \Omega \Uparrow \Theta} c u t
$$

can be eliminated in LJF and nLJF. In this latter case, $\Omega$ consists of at most one essentially right formula.

Instances of cut are divided into two categories. Key-case cuts are cuts where both cut formulas are principal, i.e., when the positive cut formula is under focus and the negative one is being decomposed. Parametric cuts refer to cuts when, in at least one subproof, the cut formula is not principal. The parametric formula can be a synchronous formula under focus or an asynchronous formula.

We first illustrate the proof in the MALLF case. As usual, we can assume that the two subproofs involved in a cut are cut-free, since we can apply the procedure to the lowestheight cuts first. The cut-elimination procedure permutes the cut above the introduction of parametric formulas until a key case is reached. By lemma 2, this holds easily for asynchronous parametric cases. But when reduction reaches the following state:

$$
\frac{\vdash: \Delta, A \Uparrow \quad \vdash: \Delta^{\prime} \Uparrow A^{\perp}}{\vdash: \Delta \Delta^{\prime} \Uparrow} c u t
$$

the left-side subproof must end in a decision rule $\left(D_{1}\right)$, which selects a formula for focus. If the formula selected for focus is the cut formula $A$, then we have a key-case cut. If $D_{1}$ selects some other formula in $\Delta$ for focus, then we have a parametric case with a positive parametric formula.

By lemmas 2 and 3, the key-case cut will have the form

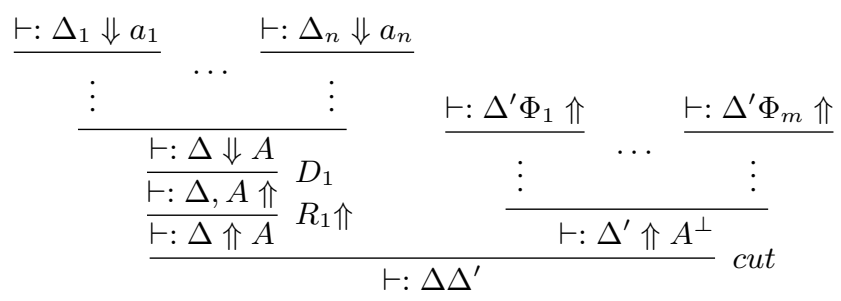

where $A \downarrow\left\{a_{1}, \ldots, a_{n}\right\}, A^{\perp} \uparrow \Phi_{i}$ for each $1 \leq i \leq m$, and $\Delta=\Delta_{1} \ldots \Delta_{n}$.

The positive parametric cut will have the form

$$
\frac{\frac{\vdash \Delta^{1} \Downarrow b_{1}}{\vdots} \ldots \frac{\vdash \Delta^{n} \Downarrow b_{n}}{\vdots}}{\frac{\frac{\vdash \Delta, A \Downarrow B}{\vdash \Delta, A, B \Uparrow} D_{1} \quad \vdash: \Delta^{\prime} \Uparrow A^{\perp}}{\vdash: B, \Delta \Delta^{\prime}} c u t}
$$

where $B \downarrow\left\{b_{1}, \ldots, b_{n}\right\}$ and $\Delta, A=\Delta^{1} \ldots \Delta^{n}$.

It is also possible that both $A$ and $A^{\perp}$ are literals, which means that the right-side subproof will continue with the form $\vdash: \Delta^{\prime}, A^{\perp} \Uparrow$, which will then also require a formula to be selected for focus. This is a critical choice-point in cut-elimination (see [9]). We must permute the cut above the subproof that contains the positive cut formula. The positive cut formula is "attractive" in the terminology of [9].

The key-case cut can be permuted into zero or more cuts involving formulas of smaller size. By lemma 4 , one of the $\Phi_{j}$ will have the form $\left\{a_{1}^{\perp}, \ldots, a_{n}^{\perp}\right\}$ (assuming $\Phi_{1}, \ldots, \Phi_{m}$ are exhaustive). For each $a_{i}$, if it is a positive literal then $\Delta_{i}=\left\{a_{i}^{\perp}\right\}$. If $a_{i}$ is negative, then by reaction $\left(R_{1} \Downarrow\right)$ we have a subproof ending in $\vdash: \Delta_{i} \Uparrow a_{i}$, to which we can apply 
an inductive hypothesis, i.e., a cut between $\vdash: \Delta_{i} \Uparrow a_{i}$ and the subproof containing $\Phi_{j}$. In either case we obtain a proof of $\vdash \Delta_{i} \Delta^{\prime}, a_{1}^{\perp} \ldots a_{i-1}^{\perp}, a_{i+1}^{\perp}, \ldots a_{n}^{\perp} \Uparrow$, and by repeated cuts (each involving a cut formula of smaller size), we get the conclusion $\vdash \Delta \Delta^{\prime} \Uparrow$.

For the parametric cut, exactly one of the $\Delta^{i}$ will contain the cut formula $A$. If $b_{i}$ is a positive literal, it cannot be the case that $b_{i}=A^{\perp}$ because $A$ is assumed positive. This critical fact relies on the choice to always permute the cut above the subproof with the positive cut formula. Thus $b_{i}$ must be negative and by $R_{1} \Downarrow$ we have a subproof of $\vdash$ : $\Delta^{i} \Uparrow b_{i}$. The original cut is permuted to a cut between $\vdash: \Delta^{i} \Uparrow b_{i}$ and $\vdash: \Delta^{\prime} \Uparrow A^{\perp}$ with a lower proof-height measure. Again by lemma 3, we can then synthesize the conclusion $\vdash: B, \Delta \Delta^{\prime}$.

The difference between cut-elimination for MALLF and for the classical LKF involves contraction (via $D_{2}$ ) and weakening (via $I_{1}$ and $I_{2}$ ). The following lemma shows that weakening and contraction are admissible in LKU.

Lemma 6: If $\vdash A, A, \Gamma: \Delta \Uparrow \Theta$ has a cut-free proof, then $\vdash A, \Gamma: \Delta \Uparrow \Theta$ has a cut-free proof of the same height. If $\vdash \Gamma: \Delta \Uparrow \Theta$ has a cut-free proof, then $\vdash A, \Gamma: \Delta \Uparrow \Theta$ has a cut-free proof of the same height.

It is important to note that the explicit contraction in $D_{2}$ is restricted to positive formulas. The key-case cut is preceded above by several parametric cuts. That is, for the sequent $\vdash A, \Gamma: \Downarrow A$, the occurrence of $A$ under focus is erased by a key-case cut while the "copy" is erased by parametric cuts. The parametric cuts have lower proof-height measures while the key cut reduces to smaller cut formulas. This argument would fail if we cannot assume that the $A$ is positive: if $A$ is negative then there would be no key case.

The LKU framework allows us to identify elements of cutelimination proofs that are "reusable." This is exemplified in the intuitionistic case. A proof of cut-elimination for LJF as originally presented is found in [12]. It involves a simultaneous induction on seven versions of cut. A much simpler proof is possible in the unified context. The principal additional work needed for the intricacies of intuitionistic polarity is captured by the following lemma, which is provable by simultaneous induction on formulas.

Lemma 7: Let $A$ be an essentially right formula and $B$ an essentially left formula. Let $\Phi_{A}, \Phi_{B}, \Psi_{A}, \Psi_{B}$, be multisets such that $A \uparrow \Phi_{A}, B \uparrow \Phi_{B}, A \downarrow \Psi_{A}$, and $B \downarrow \Psi_{B}$. Then:

$\Phi_{A}$ contains exactly one essentially right formula;

$\Psi_{B}$ contains exactly one essentially left formula;

$\Phi_{B}$ consists of only essentially left formulas; and

$\Psi_{A}$ consists of only essentially right formulas.

With this lemma, cut-elimination for LJF can be proved in essentially the same way as it is proved for other logics: by applying lemmas 2, 3, and 4. The technical argument involves a few more cases than for MALLF or LKF since both $D_{1}$ and $D_{2}$ are used.

\section{Communication Between Fragments}

Since all the fragments of LKU share the same connectives and atoms, different fragments can interact using cuts If we are only interested in cut-free classical proofs, then all cuts between fragments collapse to classical cuts. In certain circumstances, cut-elimination can preserve more structure. We give two such examples. A formula is pure with respect to a polarity if all of its subformulas have the same polarity. Focusing on purely positive formulas leads to constructive proofs.

Theorem 8: Let $A$ be a purely +2 formula and let $\Delta$ consist of purely -2 formulas. Given an LKF proof of $\vdash$ : $A, \Delta$ and a LJF proof of $\vdash \Gamma^{\perp}: \Omega \Uparrow A^{\perp}, \Theta$, the following cut:

$$
\frac{\vdash: \Uparrow A, \Delta \vdash \Gamma^{\perp}: \Omega \Uparrow A^{\perp}, \Theta}{\Gamma^{\perp}: \Omega \Uparrow \Delta \Theta} c u t
$$

can be replaced by a cut-free proof in LJF.

The proof of this theorem follows the same format as other cut-elimination proofs and uses the observation that $A$ must be selected for focus in the LKF proof.

Note that formulas such as $P \vee^{+} P^{\perp}$ are excluded from the scope of the theorem because they cannot be purely of one polarity. The scope of the theorem is expanded when one considers that, except for the quantifiers, every classical connective has an equivalent one of the opposite polarity.

Now consider cutting between a MALLF proof and an LJF proof. It is not immediate that a MALLF proof of an intuitionistic end-sequent (all formulas on the right side of $\Uparrow$ ) can be transformed into a intuitionistic proof. MALLF proofs may "split the context" differently from an intuitionistic proof.

The following lemma generalizes a key property of intuitionistic sequents that is valid in any fragment of LKU.

Lemma 9: Let $\Gamma, \Delta, \Theta$ consist of only essentially left intuitionistic formulas. There is no LKU proof of $\vdash \Gamma: \Delta \Uparrow \Theta$ that does not include an instance of the $T$ rule.

This lemma is proved by contradiction: there cannot be such a proof of minimum height. With this lemma we can show that a MALLF proof of an intuitionistic sequent can be transformed into an LJF proof. From cut-elimination in LJF, we also have the following admissible cross-cut:

Theorem 10: Given an LJF proof of $\vdash \Gamma^{\perp}: \Uparrow A$ and a MALLF proof of $\vdash$ : $A^{\perp}, B$ where $B$ is an essentially right intuitionistic formula, the following cut

$$
\frac{\vdash \Gamma^{\perp}: \Uparrow A \quad \vdash: \Uparrow A^{\perp}, B}{\vdash \Gamma^{\perp}: \Uparrow B} c u t
$$

can be replaced by a cut-free proof in LJF.

\section{A Limited ClassicAl-LineAR HybRid LOGIC}

The existence of intuitionistic logic as a hybrid logic with both linear and classical characteristics suggests that other such hybrids may also exist. It is tempting to define such a 


$$
\begin{gathered}
\frac{\vdash \Gamma: Q^{\perp} \Downarrow Q}{\vdash 1} I_{1} \frac{\vdash \Gamma: \Delta, C \Uparrow \Theta}{\vdash \Gamma: \Delta \Uparrow C, \Theta} R_{1} \uparrow \frac{\vdash \Gamma: \Delta \Uparrow N}{\vdash \Gamma: \Delta \Downarrow N} R_{1} \Downarrow \\
\frac{\vdash \Gamma: \Delta \Downarrow P}{\vdash \Gamma: \Delta, P \Uparrow} D_{1} \quad \frac{\vdash \Gamma, \mathcal{Q}^{\perp}: \Downarrow \mathcal{Q}}{\vdash \mathcal{L}} I_{2} \frac{\vdash \Gamma: \Delta \Uparrow \Theta}{\vdash \Gamma: \Delta \Uparrow \mathcal{D}, \Theta} R_{2} \Uparrow \\
\frac{\vdash \Gamma: \Uparrow \mathcal{N}}{\vdash \Gamma: \Downarrow \mathcal{N}} R_{2} \Downarrow \frac{\vdash \mathcal{P}, \Gamma: \Downarrow \mathcal{P}}{\vdash \mathcal{P}, \Gamma: \Uparrow} D_{2}
\end{gathered}
$$

$Q:+1$ atom, $C:+1$ formula or -1 atom, $N:-1$ formula, $P$ : +1 formula. $\mathcal{Q}:+2$ atom, $\mathcal{D}:+2$ formula or -2 literal, $\mathcal{N}:-2$ formula, $\mathcal{P}:+2$ formula

Figure 7. The Hybrid Logic $H L L$

logic by restricting the level- 1 structural rules to $+1 /-1$ formulas and the level- 2 rules to $+2 /-2$ formulas. Cutelimination fails, however, for such a system since it is possible to arbitrary interleaving linear and classical connectives. To illustrate this issue, consider translating formulas such as $(A \otimes B) \wedge^{+} C$ into linear logic, say, into ! $(A \otimes B) \otimes ! C$ (as suggested by the LU tables). Focusing cannot continue past the !. It would be valid to transfer from a linear focusing state to a classical one, but not vice versa. One can require that classical formulas contain no linear subformulas. Let us call this logic "High-Low Logic", or HLL. The structural rules of HLL are found in Figure 7.

Clearly, both classical logic and MALL are found as subfragments of HLL. The sample hybrid formula $A$ \& $\left(A^{\perp} \otimes\right.$ $A^{\perp}$ ) is provable if $A$ is classical but not if $A$ is linear.

It is also possible to understand HLL by a translation to linear logic. We preserve the linear connectives and translate the classical connectives as suggested by LU. For example, if $A$ is +2 and $B$ is -2 then $A \wedge^{+} B$ is translated as $A \otimes ! B$ and $A \wedge^{-} B$ is translated as ? $A \& B$.

The following cuts can be eliminated within HLL.

$$
\begin{gathered}
\frac{\vdash \Gamma: \Delta \Uparrow A, \Theta \vdash \Gamma^{\prime}: \Delta^{\prime} \Uparrow A^{\perp}, \Theta^{\prime}}{\vdash \Gamma \Gamma^{\prime}: \Delta \Delta^{\prime} \Uparrow \Theta \Theta^{\prime}} C^{\prime} t_{1} \\
\frac{\vdash \mathcal{A}, \Gamma: \Delta \Uparrow \Theta \vdash \Gamma^{\prime}: \Uparrow \mathcal{A}^{\perp}}{\vdash \Gamma \Gamma^{\prime}: \Delta \Uparrow \Theta} C u t_{2}
\end{gathered}
$$

Here, $A$ is a +1 or -1 formula and $\mathcal{A}$ is a classical formula.

To illustrate cut-reduction in HLL, assume that $\mathcal{N}$ is a -2 formula and that $M$ is a -1 formula. The proof

$$
\begin{aligned}
& \frac{\vdash \Gamma: \Uparrow \mathcal{N}}{\vdash \Gamma: \Downarrow \mathcal{N}} R_{2} \Downarrow \frac{\vdash \Gamma: \Delta \Uparrow M}{\vdash \Gamma: \Delta \Downarrow M} R_{1} \Downarrow
\end{aligned}
$$

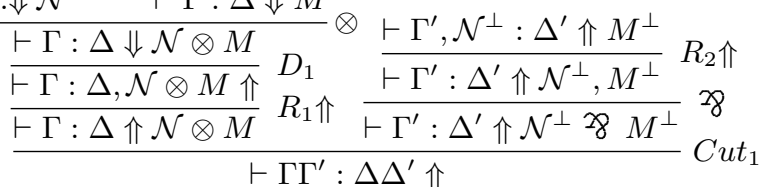

is reduced to

$$
\frac{\vdash \Gamma^{\prime}, \mathcal{N}^{\perp}: \Delta^{\prime} \Uparrow M^{\perp} \vdash \Gamma: \Uparrow \mathcal{N}}{\frac{\vdash \Gamma \Gamma^{\prime}: \Delta^{\prime} \Uparrow M^{\perp}}{\vdash \Gamma \Gamma \Gamma^{\prime}: \Delta \Delta^{\prime} \Uparrow}+\Gamma: \Delta \Uparrow M} C_{2} \quad t_{1}
$$

This example can be generalized into the core of the cutelimination proof, which shows that a key-case cut will be reduced to $\mathrm{Cut}_{2}$ rules for the classical subformulas of the cut formula and $\mathrm{Cut}_{1}$ rules for the linear subformulas.

While HLL exhibits reasonable cut-elimination properties, it also diverges from the other major fragments of LKU in significant ways. In the other fragments, provability is invariant under the assignment of different polarities to atomic formulas. This invariance fails in HLL. Switching between linear and classical atoms may affect provability, as with switching between -2 and +2 atoms. Moreover, the classical equivalences between the positive and negative versions of connectives, such as $\mathrm{V}^{-}$and $\mathrm{V}^{+}$, hold only in a purely classical context. In a mixed linear-classical context, $\mathrm{V}^{-}$is equivalent to 8 . This apparent anomaly does not contradict cut-elimination as stated above. Observe that one cannot replace a $\mathrm{V}^{-}$with a $\mathrm{V}^{+}$through cut except in a purely classical context. No admissible cut can be applied on the sequents

$$
\vdash A^{\perp} \wedge^{+} A^{\perp}: \Uparrow A \vee^{+} A \text { and } \quad \vdash: A^{\perp} \otimes A^{\perp} \Uparrow A \vee^{-} A
$$

because the linear context in the right sequent is not empty.

\section{FUtURE WORK: UnRESTRICTED Hybrid LOGIC}

Girard's LU system leaves open the possibility of mixing logics without restriction. However, designing a focused system that is entirely faithful to LU faces difficulties. For example, the De Morgan dualities fail when "neutral" formulas are mixed with classical ones.

In this paper, we have employed the different approach of carefully restricting the structure of formulas and sequents using polarity information. Extracting a focused proof system for intuitionistic logic is a powerful validation of this approach. Extracting the logic HLL is another example. One may consider another hybrid system in this same style in which the only classical connectives allowed are the additives $\mathrm{V}^{+}$and $\wedge^{-}$. In this system $A \mathrm{\vee}^{+} B$ is equivalent to ? $(A \oplus B)$ in linear logic, and unlike HLL, it would be valid to transfer from classical to linear focusing. But linear connectives may not join classical subformulas without a change in positive/negative polarity ${ }^{1}$.

Still another approach to developing hybrid logic is to extend LKU with new polarities and structural rules. Restrictions on formulas are replaced by even greater sensitivity to polarity information. Focusing can be separated into distinct levels. For example, classical focusing can be represented by

\footnotetext{
${ }^{1}$ The fact that LU's notion of classical polarity is compatible with focusing can be explained by equivalences such as $!(! A \otimes ! B) \equiv ! A \otimes ! B$ : the ! can be dropped on subformulas of the same polarity. But we also note the equivalences ? $(? \oplus ? B) \equiv ?(A \oplus B)$ and ? $\exists x . ? A \equiv ? \exists x . A$, as well as their duals by negation. Unlike the LU equivalences, these apply only to the additives. Structural rules on a formula need only be applied at the outset, not to subformulas, thus enabling hybrid focusing.
} 
$\Downarrow^{2}$ and linear focusing by $\Downarrow^{1}$. Transition between focusing modes can be formulated by lateral reaction rules such as

$$
\frac{\vdash \Gamma: \Delta \Downarrow^{2} A}{\vdash \Gamma: \Delta \Downarrow^{1} A} L \Downarrow
$$

where $A$ is a classical formula. More flexible variants of $R \Uparrow$ are also needed, including those that insert asynchronous formulas into the classical context, as one would expect from a system with the full power of linear logic. We are, in fact, currently studying a system with three distinct types of $\Downarrow$ and three of $\Uparrow$.

\section{ACKNOWLEDGMENT}

This work has been supported by INRIA through the "Equipes Associées" Slimmer.

\section{REFERENCES}

[1] J.-Y. Girard, "On the unity of logic," Annals of Pure and Applied Logic, vol. 59, pp. 201-217, 1993.

[2] H. Nakano, "A constructive logic behind the catch/throw mechanism," Annals of Pure and Applied Logic, vol. 69, no. 2-3, pp. 269-301, 1994.

[3] D. Miller, G. Nadathur, F. Pfenning, and A. Scedrov, "Uniform proofs as a foundation for logic programming," Annals of Pure and Applied Logic, vol. 51, pp. 125-157, 1991.

[4] J.-M. Andreoli, "Logic programming with focusing proofs in linear logic," J. of Logic and Computation, vol. 2, no. 3, pp. 297-347, 1992.

[5] K. Chaudhuri, D. Miller, and A. Saurin, "Canonical sequent proofs via multi-focusing," in Fifth International Conference on Theoretical Computer Science, ser. IFIP, G. Ausiello, J. Karhumäki, G. Mauri, and L. Ong, Eds., vol. 273. Springer, Sep. 2008, pp. 383-396.
[6] D. Miller and V. Nigam, "Incorporating tables into proofs," in CSL 2007: Computer Science Logic, LNCS 4646, J. Duparc and T. A. Henzinger, Eds. Springer, 2007, pp. 466-480.

[7] H. Herbelin, "Séquents qu'on calcule: de l'interprétation du calcul des séquents comme calcul de lambda-termes et comme calcul de stratégies gagnantes," Ph.D. dissertation, Université Paris 7, 1995.

[8] R. Dyckhoff and S. Lengrand, "LJQ: a strongly focused calculus for intuitionistic logic," in Computability in Europe 2006, LNCS 3988, A. Beckman, et al, Ed.Springer, 2006, pp. $173-185$.

[9] V. Danos, J.-B. Joinet, and H. Schellinx, "A new deconstructive logic: Linear logic," Journal of Symbolic Logic, vol. 62, no. 3, pp. 755-807, 1997.

[10] R. Jagadeesan, G. Nadathur, and V. Saraswat, "Testing concurrent systems: An interpretation of intuitionistic logic," in FSTTCS 2005: Foundations of Software Technology and Theoretical Computer Science, LNCS 3821. Hyderabad, India: Springer, 2005.

[11] O. Laurent, M. Quatrini, and L. T. de Falco, "Polarized and focalized linear and classical proofs," Ann. Pure Appl. Logic, vol. 134, no. 2-3, pp. 217-264, 2005.

[12] C. Liang and D. Miller, "Focusing and polarization in intuitionistic logic," in CSL 2007: Computer Science Logic, LNCS 4646, J. Duparc and T. A. Henzinger, Eds. Springer, 2007, pp. 451-465.

[13] J.-Y. Girard, "A new constructive logic: classical logic," Math. Structures in Comp. Science, vol. 1, pp. 255-296, 1991.

[14] G. Gentzen, "Investigations into logical deductions," in The Collected Papers of Gerhard Gentzen, M. E. Szabo, Ed. Amsterdam: North-Holland, 1969, pp. 68-131.

[15] O. Laurent, "Etude de la polarisation en logique," Thèse de Doctorat, Université Aix-Marseille II, Mar. 2002. 